\title{
Quasi-Phase-Matching High-Harmonic Radiation Using Chirped THz Pulses
}

\author{
Katalin Kovács, ${ }^{1,2, *}$ Emeric Balogh, ${ }^{1}$ János Hebling, ${ }^{3}$ Valer Toşa, ${ }^{2}$ and Katalin Varjú ${ }^{1}$ \\ ${ }^{1}$ Department of Optics and Quantum Electronics, University of Szeged, HU-6720 Szeged, Hungary \\ ${ }^{2}$ National Institute for R\&D of Isotopic and Molecular Technologies, RO-400293 Cluj-Napoca, Romania \\ ${ }^{3}$ Department of Experimental Physics, University of Pécs, HU-7624 Pécs, Hungary
}

(Received 7 November 2011; published 8 May 2012)

\begin{abstract}
High-order harmonic generation in the presence of a chirped THz pulse is investigated numerically with a complete 3D nonadiabatic model. The assisting $\mathrm{THz}$ pulse illuminates the high-order harmonic generation gas cell laterally inducing quasi-phase-matching. We demonstrate that it is possible to compensate the phase mismatch during propagation and extend the macroscopic cutoff of a propagated strong IR pulse to the single-dipole cutoff. We obtain 2 orders of magnitude increase in the harmonic efficiency of cutoff harmonics $(\approx 170 \mathrm{eV})$ using a THz pulse of constant wavelength, and a further factor of 3 enhancement when a chirped $\mathrm{THz}$ pulse is used.
\end{abstract}

DOI: 10.1103/PhysRevLett.108.193903

PACS numbers: 42.65.Ky, 42.65.Re, 52.38.Ph

High-order harmonic generation (HHG) in a noble gas medium is currently the widely used method to produce coherent radiation in the XUV and soft-X-ray regime. The elementary laser-atom interaction leading to HHG is understood via the three-step model [1], but the complete description of the HHG process requires considering the elementary laser-atom interaction together with the macroscopic aspects of laser and harmonic field propagation in the ionized gaseous medium. The temporal, spectral, and spatial distortions of the fundamental pulse during propagation result in a varying intensity and phase which strongly influence the produced harmonic radiation on both the single-atom and the macroscopic level [2]. The ultimate goal in an experiment is to obtain intense, coherent XUV or soft-X-ray radiation which in turn are useful in the designed applications like molecular imaging or pumpprobe experiments [3].

In Fig. 1 we show a typical case of phase mismatch (PMM) of the harmonic radiation along the HHG cell manifesting in a quasiperiodic oscillation of the harmonic intensity named Maker fringes [4]. In order to improve the phase-matching (PM) condition, a number of quasi-phasematching (QPM) techniques have been developed to at least partially compensate for the PMM arising during propagation between the source and generated harmonics. There are currently several typical QPM techniques relying either on periodic modulation of the generating field strength: propagation in diameter-modulated capillaries [5]; HHG assisted by a periodic static electric field (or other periodic waveform, e.g., sawtooth) [6,7]; QPM induced using two counter-propagating pulses [8,9]; QPM through multimode excitation in a waveguide [10]. An alternative QPM arrangement relies on periodic off switching of generation in a multijet configuration $[11,12]$.

We present here a new scheme, inspired by the idea described in [6] where the HHG gas cell was placed in a static electric field periodically modulated along the propagation direction; a pulsed $\mathrm{CO}_{2}$ laser and an amplitude mask were proposed to create $\approx 7 \mathrm{MV} / \mathrm{cm}$ static electric field periodically distributed along the HHG cell. In our opinion, this configuration was unrealistic because: (1) the $\mathrm{CO}_{2}$ laser pulse with $\approx 10 \mu \mathrm{m}$ wavelength cannot be considered as a static electric field when compared to the $\approx 100 \mu \mathrm{m}$ mask periodicity ( $2 \mathrm{~mm}$ cell length), and (2) producing a $7 \mathrm{MV} / \mathrm{cm}$ static electric field is not feasible experimentally. Yet, it is possible to overcome the shortcomings of the QPM configuration presented in [6]: we use a $\mathrm{THz}$ field imposing a proper time evolution to create QPM conditions. The advantages of this new scheme are multiple: strong $\mathrm{THz}$ pulses can be generated by optical rectification of femtosecond laser pulses, and as such the resulted $\mathrm{THz}$ pulse is inherently synchronized in time with the laser pulse generating the harmonics; fine control in space and time is available by tuning the wavelength, peak amplitude, chirp rate, and initial phase, thus the shape of the $\mathrm{THz}$ pulse can be adapted in order to optimize QPM and harmonic yield, and there is no need to

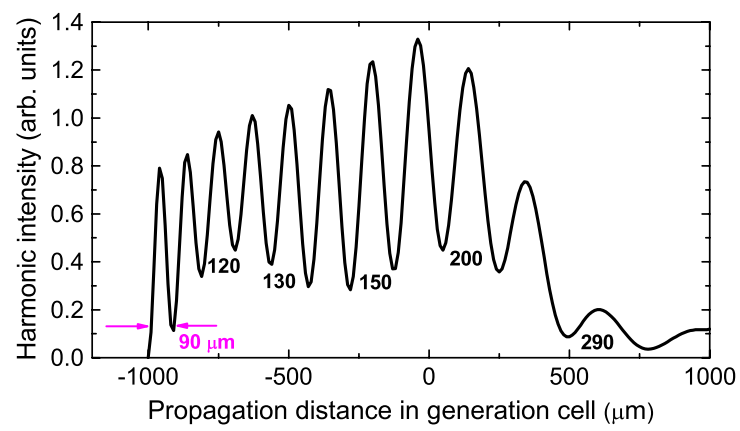

FIG. 1 (color online). On-axis variation of the H111 along the propagation direction generated by the IR pulse. The width of several Maker fringes $=2 L_{\mathrm{coh}}$ is indicated in $\mu \mathrm{m}$, other parameters are in the text. 
apply any amplitude mask. We show a schematic representation of a possible geometric configuration in Fig. 2.

The $\mathrm{THz}$ field modifies the electron trajectories and hence the single-atom dipole phase (depending on return time and action). As the generating IR pulse propagates along the gas cell, the temporal variation of the $\mathrm{THz}$ pulse, which is simultaneously present with the IR, translates to a spatial modulation of the HHG conditions along $z$. According to our results presented below, assisting pulses of a few (or few tens) $\mathrm{THz}$ frequency and a few MV/cm peak electric field are required for the enhancement of HHG. In this wavelength range, the highest electric field already presented is $1.2 \mathrm{MV} / \mathrm{cm}$ [13] achieved with $\mathrm{THz}$ pulses having $1 \mu \mathrm{J}$ energy obtained using a tilted pulse front setup [14]. Recently THz pulses with $125 \mu \mathrm{J}$ energy were generated and production of $\mathrm{THz}$ pulses with more than $10 \mathrm{~mJ}$ were predicted [15]. These were single-cycle $\mathrm{THz}$ pulses, but it is expected that using quasisinusoidal intensity modulated pump laser pulses [16] multicycle $\mathrm{THz}$ pulses with similar energy can be generated resulting up to $10 \mathrm{MV} / \mathrm{cm}$ field strength in $10 \mathrm{ps}$ long $\mathrm{THz}$ pulse on minimum $3 \mathrm{~mm}$ diameter spot.

The numerical model in which the THz-assisted HHG generation is implemented is based on a complete 3D nonadiabatic model [17], further developed to two-color HHG using arbitrary wavelength copropagating pulses [18]. The THz-assisted HHG has also been studied in the configuration where the two pulses propagate in the same direction [19]. For the current work the model has been extended to describe pulses propagating perpendicularly. (i) The propagation equation for the IR pulse is solved accounting for diffraction, dispersion (on neutrals and plasma), absorption and nonlinear Kerr effect; (ii) nonlinear dipole response is calculated in the strongfield approximation [20]; (iii) the propagation equation for the generated harmonics is solved. In order to gain a more intuitive insight into the physics behind the effect of the modulating THz pulse, saddle-point calculations were also carried out providing the phases of the single-atom dipole and the total harmonic field.

We present a case study to demonstrate the new QPM scheme with THz-assisted HHG. We start from a standard

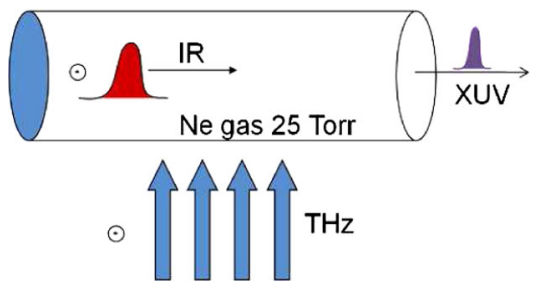

FIG. 2 (color online). Schematic representation of the configuration where the assisting $\mathrm{THz}$ pulse propagates in perpendicular direction relative to the main IR beam which propagates from left to right. Both fields are linearly polarized in the direction perpendicular to the figure plane.
HHG system by an IR pulse while looking for the optimal configuration of the $\mathrm{THz}$ pulse which induces the best possible QPM in a selected harmonic range. The particular conditions for the HHG are the following: the IR pulse has $800 \mathrm{~nm}$ central wavelength, 20 fs pulse duration, $0.2 \mathrm{~mJ}$ pulse energy, focused with a $20 \mathrm{~cm}$ focal length mirror resulting in $8 \times 10^{14} \mathrm{~W} / \mathrm{cm}^{2}$ peak intensity and $25 \mu \mathrm{m}$ beam waist. The HHG cell is $2 \mathrm{~mm}$ long filled with 25 Torr $\mathrm{Ne}$ and begins $1 \mathrm{~mm}$ before the laser focus. All distances throughout this paper are measured from the laser focus. The single-dipole cutoff order is $q=111$ (based on the relation $I_{p}+3.17 U_{p}$ ) corresponding to $172 \mathrm{eV}$ and QPM is optimized for this spectral range.

Figure 1 illustrates the on-axis intensity variation of the 111th harmonic (H111) along the propagation axis as generated with the $800 \mathrm{~nm}$ pulse alone. We can see the experimentally observable high-contrast Maker fringes, a typical result of PMM in nonlinear phenomena. On the figure we also observe that PM conditions vary along the cell, the main causes being the intensity and phase changes of the generating pulse as it propagates. In particular, the width of the fringes increases from 90 to $290 \mu \mathrm{m}$ during $2 \mathrm{~mm}$ of propagation. The actual period of the highharmonic intensity modulation and its variation is determined by the focusing conditions of the IR pulse. We use the THz field to modulate HHG conditions along the cell and compensate for PMM. We note here that obviously, looser focusing provides longer coherence lengths, but the here proposed arrangement can be equivalently applied for longer cells with longer $\mathrm{THz}$ wavelengths.

From the results shown in Fig. 1 it is straightforward that for optimal QPM the wavelength of the modulation needs to be varied, a requirement that has been observed in [21]. We therefore aim at producing a quasiperiodic spatial modulation of the generating conditions via the temporal modulation of the wavelength (chirp) of our THz pulse. A QPM effect of the perpendicularly propagating $\mathrm{THz}$ field is expected when the modulation caused by the field matches the Maker fringes observed in the IR-only case [6]. To verify this concept, we studied the effect of a THz field of fixed wavelength (120 $\mu \mathrm{m}$, corresponding to $2.5 \mathrm{THz})$ on the HHG yield shown in Fig. 3(b) with the blue dashed curve. The black solid curve in the top part of the figure illustrates the oscillation of harmonic intensity for the IR-only case replotted from Fig. 1 in the same arbitrary units. We note here, that the THz amplitude and delay have been chosen appropriately.

For more efficient QPM we use a chirped $\mathrm{THz}$ pulse such that the wavelength variation of the $\mathrm{THz}$ pulse along the cell best matches the fringe structure of the chosen harmonic: $\lambda_{\mathrm{THz}}(z) \approx 2 L_{\mathrm{coh}}(z)$. In this case study, we designed a linearly chirped $\mathrm{THz}$ pulse that follows nicely the field oscillations, as illustrated in Fig. 3(a) with the green dashed curve. The parameters of the perpendicular $\mathrm{THz}$ field are: Gaussian pulse profile with $110 \mu \mathrm{m}$ initial 


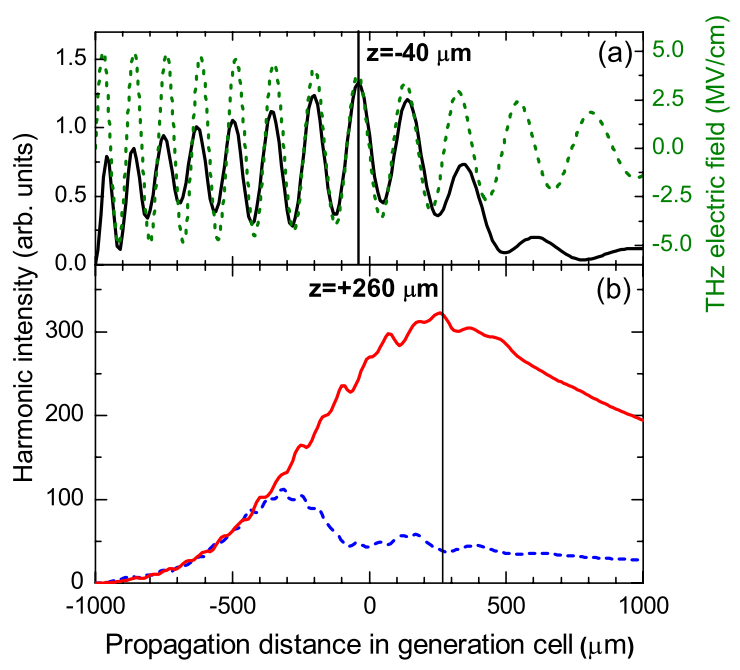

FIG. 3 (color online). (a) Black solid line: THz-free variation of $\mathrm{H} 111$ intensity. Green dotted line: $E_{\mathrm{THz}}(z)$ visualizes the correspondence with the $2 L_{\mathrm{coh}}(z)$. (b) Red solid line: On-axis variation of the H111 intensity along the propagation direction when a chirped $\mathrm{THz}$ pulse is applied. Blue dashed line: Same when the $\mathrm{THz}$ pulse has constant $\lambda=120 \mu \mathrm{m}$. Vertical lines indicate the positions of maximum harmonic yield.

wavelength, $-9 \times 10^{-7} \mathrm{fs}^{-2}$ chirp rate resulting $180 \mu \mathrm{m}$ average wavelength, $5 \mathrm{MV} / \mathrm{cm}$ peak amplitude, $8 \mathrm{ps} \mathrm{du}-$ ration, and $4 \mathrm{~mm}$ beam waist. For these parameters the red solid line in Fig. 3(b) indicates very efficient QPM for $\mathrm{H} 111$ up to $\approx 1.2 \mathrm{~mm}$ in the cell. We note here that at the end of the cell H111 emission is reduced due to divergence and plasma defocusing of the beam, and the THz field walks off the H111 IR-only intensity modulation pattern leading to loss of QPM. Comparing the highest intensities along the cell obtained with and without the $\mathrm{THz}$ field (realizable experimentally by shortening the cell or moving the focus position) shows $\mathrm{a} \approx 300$ times yield enhancement.

Optically ionized electrons traveling in the laser field are responsible for HHG, thus both amplitude and phase of the high harmonics are very sensitive to the exact shape of the electric field. The total harmonic field along $z$ is the sum of successive dipole emissions:

$$
E_{q}(z) e^{i \phi_{q}(z)}=\int_{0}^{z} \mathbf{d}_{q}^{z}\left(z^{\prime}\right) d z^{\prime}=\int_{0}^{z} a_{q}\left(z^{\prime}\right) e^{i \varphi_{q}\left(z^{\prime}\right)} d z^{\prime},
$$

where $E_{q}(z)$ and $\phi_{q}(z)$ are amplitude and phase of the qth order $\mathrm{HH}$ field accumulated till $z$, $\mathbf{d}$ is the qth Fourier component of the dipole moment vector, and the dipole phase $\varphi_{q}(z)=q \varphi_{\mathrm{IR}}(z)+\Phi_{\mathrm{at}}(z)$ is described in a traveling frame moving with $c$. The applied $\mathrm{THz}$ pulse amplitude is weak compared to the generating IR $(<1 \%)$, thus its main effect is the modulation of the accumulated phase of the electron while traveling in the field $\left[\Phi_{\mathrm{at}}(z)\right]$. Efficient QPM is explained at the harmonic dipole level. In particular, for
H111 the dipole amplitude variation $a_{q}(z)$ did not show any correlation with $E_{q}(z)$, instead we found strong correlation with the phase. Using saddle-point calculations for shortto-cutoff trajectories in the central half-cycle of the IR pulse we studied the $\varphi_{q}(z)$ and $\phi_{q}(z)$ variations, and compared the phase difference $\phi_{q}(z)-\varphi_{q}(z)$ with the total harmonic intensity $\left|E_{q}(z)\right|^{2}$. The results are shown in Fig. 4 for the central part of the cell when the HH field is built with IR pulse only (a), (c) and when assisted with chirped $\mathrm{THz}$ pulse (b), (d). As expected, while $\left|\phi_{q}(z)-\varphi_{q}(z)\right|<\pi / 2$ the successive dipole emissions add up constructively and result in an overall increase of the total harmonic field. The effect of the THz field is that it modulates the dipole phase such to keep $\left|\phi_{q}(z)-\varphi_{q}(z)\right|$ within $\pi / 2$ over longer propagation distances and pass through destructive zones in much shorter regions.

In Fig. 5 we compare harmonic spectra, on logarithmic scale, obtained in different conditions. The black line indicates the spectrum produced in a very thin $(20 \mu \mathrm{m})$ target with the IR pulse alone, which gives us practically the single-atom spectrum, with the expected cutoff located at H111. The conversion efficiency is very low due to the short cell. The red line indicates the spectrum produced by the IR pulse recorded at the position in cell where we obtained the highest $\mathrm{H} 111$ intensity, i.e., $-40 \mu \mathrm{m}$ [cf. Figure 3(a)]. We observe that harmonics in the lower plateau (below $110 \mathrm{eV}$ ) experience an increase $\left(\approx 10^{2}\right.$ times) with cell length, as more atoms contribute to the HHG process, and apparently phase matching is favorable. For the higher plateau and cutoff harmonics we observe no yield enhancement with increasing cell length, which is explained by the PMM discussed in detail for H111.

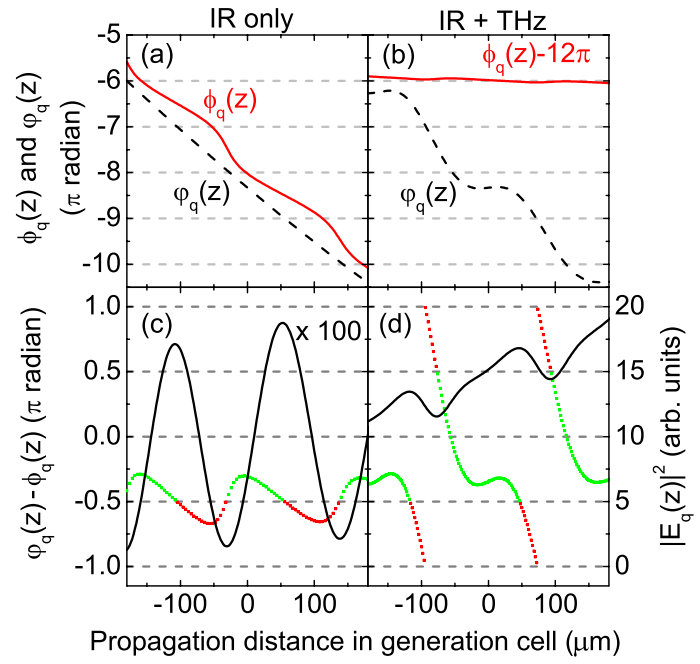

FIG. 4 (color online). Variation of the H111 dipole phase and total harmonic phase without (a) and with $\mathrm{THz}$ field present (b). Red-green dots: $\varphi_{q}(z)-\phi_{q}(z)$ phase difference with scale on the left. Black line: H111 intensity $\left|E_{q}(z)\right|^{2}$, scale on the right. (c) IR-only case; (d) THz-assisted case. 


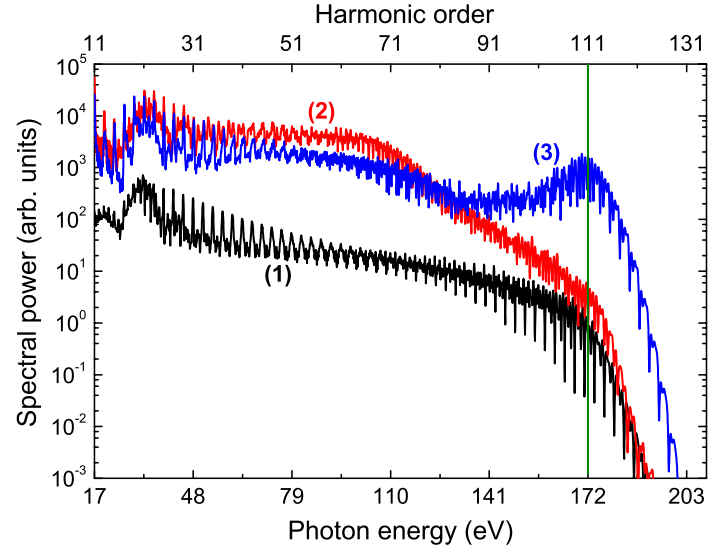

FIG. 5 (color online). Logarithmic plot of HH spectra. Black (1): harmonic spectrum generated with IR-only after $20 \mu \mathrm{m}$ propagation. Red (2): IR-only spectrum at the position of max. H111 intensity $(-40 \mu \mathrm{m})$. Blue (3): spectrum obtained with THz-assisted HHG at the position of max. H111 intensity $(+260 \mu \mathrm{m})$. THz pulse was linearly chirped such to best match the on-axis periodicity of H111 (172 eV). Vertical line: position of H111.

The blue line depicts the spectrum obtained with the chirped $\mathrm{THz}$ pulse assisting the HHG process at the position in the cell where the H111 intensity reaches its maximum $(+260 \mu \mathrm{m})$. Since the THz parameters were chosen to optimize QPM for H111, the spectrum is enhanced in that range. At $\mathrm{H} 111$ we obtain $\approx 1060$ times increase in the maximum harmonic yield after $\approx 1200 \mu \mathrm{m}$ propagation compared to the yield at $20 \mu \mathrm{m}$. With perfect PM and constant emission rate the harmonic yield should have increased quadratically with propagation distance, resulting in $\mathrm{a} \approx 3600$ times enhancement. Our QPM method reaches $\approx 30 \%$ of this value while the emission rate is decreasing. The effective harmonic enhancement in the selected spectral region is $\approx 100$ times using constant $120 \mu \mathrm{m} \mathrm{THz}$ wavelength, and $\approx 300$ times with chirped $\mathrm{THz}$ assistance.

In the present arrangement, the $\mathrm{THz}$ pulse is too weak to increase the single-atom cutoff. For that purpose, orders of magnitude higher $\mathrm{THz}$ field strength is required $[19,22]$. Here we induce QPM conditions to restore the single-atom cutoff and in addition to enhance the yield by around 300 times compared to the $\mathrm{THz}$-free case.

In the geometry sketched in Fig. 2 for the used THz field strength and spot size, we need $70 \mathrm{~mJ} \mathrm{THz}$ pulse energy which is more than 2 orders of magnitude larger than available now in one-cycle THz pulse [15]. However, using cylindrical focusing one can reduce the vertical $\mathrm{THz}$ spot size to $0.5 \mathrm{~mm}$ which lowers the needed $\mathrm{THz}$ energy by a factor of $\approx 10$. By further optimization of the beam waist and the focusing in the cell the needed THz energy might be reduced to $\approx 4 \mathrm{~mJ}$. Creating the $\mathrm{THz}$ field with the standing wave produced by two counter-propagating beams the needed energy can be reduced to $\approx 2 \times 1 \mathrm{~mJ}$.
Further investigation is going on to demonstrate that the presented QPM configuration is flexible and that by tuning $\mathrm{THz}$ pulse parameters one can select the spectral range in which harmonic radiation can be amplified. Our results demonstrate that by tuning the $\mathrm{THz}$ field one can finely shift the central frequency of the amplified spectral range toward lower harmonics. Another key is the IR pulse energy which shifts the HH cutoff. For our case, a $\pm 25 \%$ IR pulse energy variation combined with appropriate $\mathrm{THz}$ field allowed amplification in the 140-220 eV spectral domain. This might be of central importance in seeding free electron lasers in this range of photon energies. Recently mid-IR laser pulses are being also used for HHG in order to achieve higher cutoff energy. As the harmonic yield scales with $\lambda^{-5.5}$ [23], an efficient QPM is even more important for this domain. Applying the THzassisted QPM scheme could significantly increase the harmonic yield at selected spectral ranges approaching the water-window.

The authors acknowledge the support of: TÁMOP-4.2.1/ B-09/1/KONV-2010-0005-Creating the Center of Excellence at the University of Szeged supported by the EU and cofinanced by the European Social Fund, CNCSUEFISCDI Project No. PN-II-RU-PD-2011-3-0236 (K. K.); FP7 Contract No. ITN-2008-238362 (ATTOFEL) (E. B.); Hungarian research Grant OTKA 81364, and the Bolyai Foundation (K. V.). We thank NIRDIMT Data Center and NIIF Institute for computation time.

*kkovacs@itim-cj.ro

[1] P. B. Corkum, Phys. Rev. Lett. 71, 1994 (1993).

[2] M. B. Gaarde, J. L. Tate, and K. J. Schafer, J. Phys. B 41, 132001 (2008).

[3] F. Krausz and M. Ivanov, Rev. Mod. Phys. 81, 163 (2009).

[4] S. Kazamias, D. Douillet, C. Valentin, F. Weihe, F. Augé, Th. Lefrou, G. Grillon, S. Sebban, and Ph. Balcou, Phys. Rev. A 68, 033819 (2003).

[5] A. Paul, R. A. Bartels, R. Tobey, H. Green, S. Weiman, I. P. Christov, M. M. Murnane, H. C. Kapteyn, and S. Backus, Nature (London) 421, 51 (2003).

[6] C. Serrat and J. Biegert, Phys. Rev. Lett. 104, 073901 (2010).

[7] P. Sidorenko, M. Kozlov, A. Bahabad, T. Popmintchev, M. Murnane, H. Kapteyn, and O. Cohen, Opt. Express 18, 22686 (2010).

[8] X. Zhang, A. L. Lytle, T. Popmintchev, X. Zhou, H. C. Kapteyn, M. M. Murnane, and O. Cohen, Nature Phys. 3, 270 (2007).

[9] A. L. Lytle, X. Zhang, P. Arpin, O. Cohen, M. M. Murnane, and H. C. Kapteyn, Opt. Lett. 33, 174 (2008).

[10] M. Zepf, B. Dromey, M. Landreman, P. Foster, and S. M. Hooker, Phys. Rev. Lett. 99, 143901 (2007).

[11] J. Seres, V.S. Yakovlev, E. Seres, C. Streli, P. Wobrauschek, C. Spielmann, and F. Krausz, Nature Phys. 3, 878 (2007). 
[12] V. Tosa, V. S. Yakovlev, and F. Krausz, New J. Phys. 10, 025016 (2008).

[13] H. Hirori, A. Doi, F. Blanchard, and K. Tanaka, Appl. Phys. Lett. 98, 091106 (2011).

[14] J. Hebling, G. Almasi, I. Kozma, and J. Kuhl, Opt. Express 10, 1161 (2002), http://www.opticsinfobase.org/oe/ abstract.cfm?URI=oe-10-21-1161.

[15] J. A. Fülöp, L. Pálfalvi, M. C. Hoffmann, and J. Hebling, Opt. Express 19, 15090 (2011); J. A. Fülöp, L. Pálfalvi, S. Klingebiel, G. Almási, F. Krausz, S. Karsch, and J. Hebling, Opt. Lett. 37, 557 (2012).

[16] Z. Chen, X. Zhou, C. A. Werley, and K. A. Nelson, Appl. Phys. Lett. 99, 071102 (2011).

[17] E. Takahashi, V. Tosa, Y. Nabekawa, and K. Midorikawa, Phys. Rev. A 68, 023808 (2003).
[18] V. Tosa, C. Altucci, K. Kovacs, M. Negro, S. Stagira, C. Vozzi, and C. Velotta, IEEE J. Sel. Top. Quantum Electron., 18239 (2012).

[19] E. Balogh, K. Kovacs, P. Dombi, J. A. Fulop, G. Farkas, J. Hebling, V. Tosa, and K. Varju, Phys. Rev. A 84, 023806 (2011).

[20] M. Lewenstein, P. Balcou, M. Y. Ivanov, A. L. Huillier, and P. B. Corkum, Phys. Rev. A 49, 2117 (1994).

[21] T. Robinson et al., in Ultrafast Phenomena XVI, Series in Chemical Physics Vol. 92 (Springer, New York, 2009), pp. $15-17$.

[22] W. Hong, P. Lu, P. Lan, Q. Zhang, and X. Wang, Opt. Express 17, 5139 (2009).

[23] J. Tate, T. Auguste, H. G. Muller, P. Salières, P. Agostini, and L. F. DiMauro, Phys. Rev. Lett. 98, 013901 (2007). 